\title{
Conceptual design and optimization of a back- scatter 14-MeV neutron time-of-flight spectrometer for fusion applications
}

\author{
Göran Ericsson ${ }^{1}$ \\ Department of Physics and Astronomy, Uppsala University, \\ Uppsala, Sweden \\ E-mail: goran.ericsson@physics.uu.se
}

\author{
N. Dzysiuk, E. Andersson Sundén, S. Conroy, C. Hellesen, A. Hjalmarsson, \\ M. Skiba \\ Department of Physics and Astronomy, Uppsala University, \\ Uppsala, Sweden
}

\begin{abstract}
The time-of-flight technique is a well-established and successful diagnostic tool for neutron energy measurements. In fusion research, neutron time-of-flight systems have primarily been used for the emission around $\mathrm{E}_{\mathrm{n}}=2.5 \mathrm{MeV}$ in $\mathrm{D}$ plasma operations. The normal implementation of the time-of-flight technique, using forward scattering of the incoming neutrons in elastic n,p collisions has in general not been deemed suitable for diagnosing the emission around $14 \mathrm{MeV}$ in DT plasmas, due to the large size and complexity required of such systems. This paper deals with the design of a back-scatter timeof-flight (bTOF) spectrometer as a possible instrument for performing high resolution measurements of $14 \mathrm{MeV}$ fusion neutrons. The instrument is based on two sets of scintillators, a first set exposed to a collimated beam of neutrons from the plasma and a second detector set placed in the backward (up-stream) direction. The scintillators of the first set are enriched in deuterium to achieve neutron backscattering. Here we present a preliminary design of a high-resolution bTOF instrument of optimal geometry suitable for ITER. The results have been obtained by Monte Carlo simulations based on the MCNPX code. The system conceptual design and preliminary performance indicators in terms of efficiency and resolution as well as background subtraction possibilities are presented.
\end{abstract}

\footnotetext{
${ }^{1}$ Presenter 
Conceptual design and optimization of a back-scatter 14-MeV neutron time-of-flight spectrometer ...

First EPS Conference on Plasma Diagnostics - $1^{\text {st }}$ ECPD 14-17 April 2015,

Villa Mondragone , Frascati (Rome) Italy 


\section{Introduction}

In this work we present results from the optimization of a backscatter time-of-flight neutron spectrometer suitable for ITER. In fusion applications, neutrons are produced in the very fusion reactions themselves:

$$
\begin{aligned}
& \mathrm{D}+\mathrm{D} \rightarrow{ }^{3} \mathrm{He}+\mathrm{n} \quad\left(\mathrm{E}_{\mathrm{n}}=2.45 \mathrm{MeV}\right) \\
& \mathrm{D}+\mathrm{T} \rightarrow{ }^{4} \mathrm{He}+\mathrm{n} \quad\left(\mathrm{E}_{\mathrm{n}}=14.0 \mathrm{MeV}\right)
\end{aligned}
$$

The neutrons escape the magnetically confined plasma and can be detected outside of the fusion device. They can provide information on a wide range of performance and plasma parameters: $Y_{n}, P_{\text {fus }}, T_{i}, v_{\text {rot }}$, thermal fraction, details of the fuel ion distribution function, etc.

For this reason, neutron diagnostics is routinely used on most high performance fusion experiments in the form of counters, cameras and spectrometers. Neutron spectrometry can provide detailed information on the state of the fusion fuel ions ( $\mathrm{D}$ and $\mathrm{T}$ ).

\section{The backscatter time-of-flight principle}

Time-of-flight is a successful neutron spectrometry technique in many applications. In fusion, due to the continuous character of the neutron emission, it is implemented as doublescattering instruments, measuring the time of travel for a neutron over a known distance between a "start" and a "stop" detector. Forward TOF instruments have been implemented at several fusion experiments and the state-of-the-art is represented by the TOFOR [1] at JET, optimized for measurements of fusion neutrons around $2.45 \mathrm{MeV}$ in D plasma operations.

For 14-MeV measurements the forward-TOF technique is less used, due to the large size and complexity of the instrument. However, a possibility exists to use the TOF technique in a backward scattering configuration [2], thereby preserving many of the advantages of the TOF method while still limiting size and complexity. In this work we have investigated an optimized geometry for a backscatter TOF instrument.

Such a backscatter time-of-flight instrument (bTOF) is based on the use of a deuterated scintillator as first scatterer (D1) [3]. A schematic side view is shown in Fig. 1a. Here, a collimated beam of neutrons enter from below and impinges of the segmented D1 deuterated scintillator ("start"). Some neutrons undergo elastic n,d scattering, which gives (some) neutron recoils in the backward direction. Some of the backscattered neutrons are subsequently detected in the segmented D2 detector, made of ordinary scintillator material ("stop”).

There are several advantages associated with the bTOF principle:

- The recoil deuteron in the D1 detector is of high energy and hence gives rise to a large "start" scintillator signal.

- The energy of the backscattered neutron is low enough to give a long neutron flight time even for fairly short D1-D2 distances.

- The energy of the backscattered neutron is high enough to give a sufficiently large D2 scintillator signal - it is comparable with TOFOR in D operations.

- The differential cross section of n,d scattering is strongly peaked in the backward (and forward) direction, see Fig. 1b), giving high efficiency. 

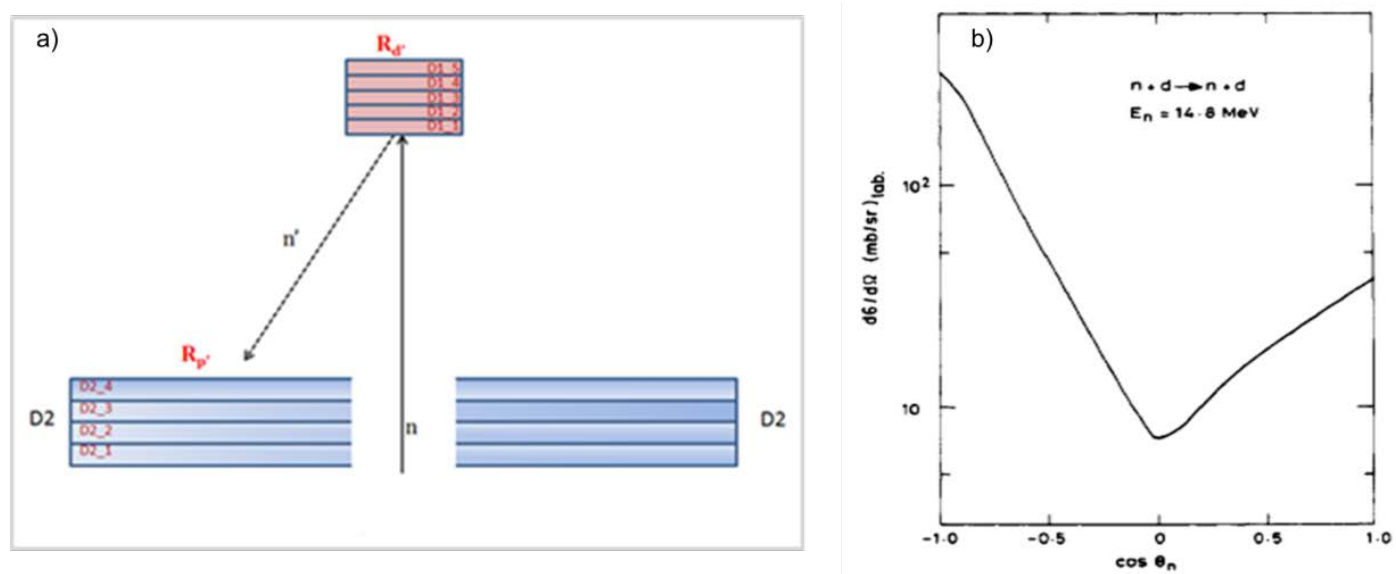

Fig. 1 a) Schematic of a backscatter TOF instrument. b) The differential cross section of $\mathrm{n}+\mathrm{d} \rightarrow \mathrm{n}+\mathrm{d}$ elastic scattering in the laboratory system

\section{MCNPX modeling}

A graphical representation of the MCNPX model used in this work is shown in Fig. 2.

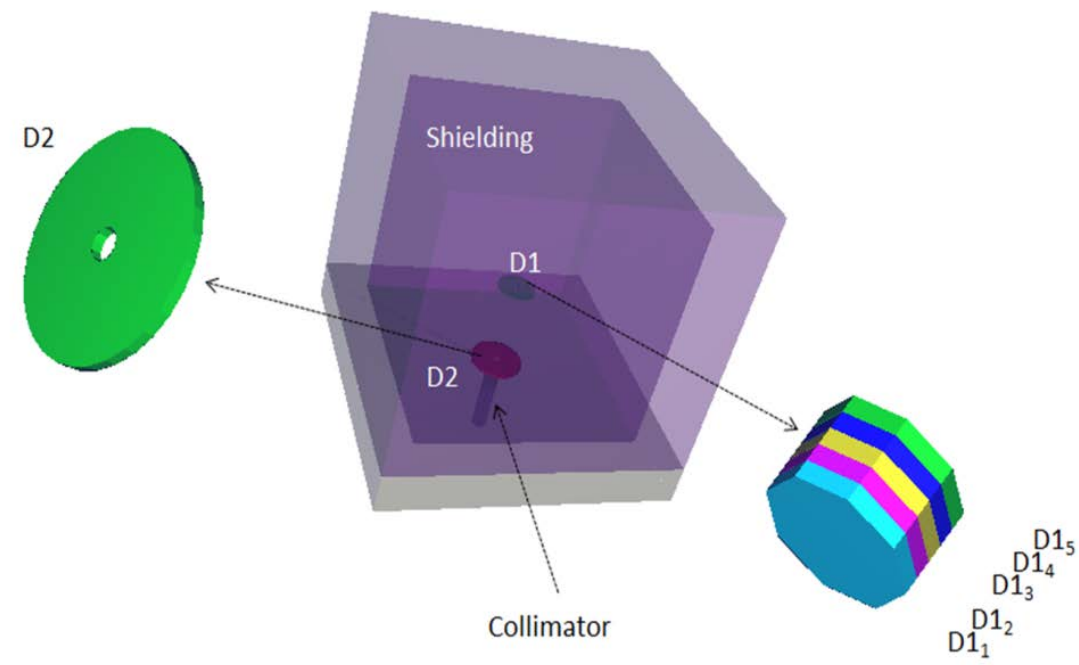

Fig. 2 The 3D MCNPX model of a bTOF spectrometer.

The main components of the MCNPX model are:

- A concrete radiation shield surrounding the whole installation.

- A circular neutron collimator forming the emitted plasma neutrons into a "neutron beam".

- A segmented, circular $(\mathrm{R}=20 \mathrm{~mm}) \mathrm{D1}$ deuterated scintillator - first scatterer - placed in the collimated neutron beam.

- An annular, circular D2 ordinary scintillator - second scatterer - placed upstream of D1, outside of the neutron beam. 
The material composition of the D1 is (relative number densities) C (50), $\mathrm{D}(47.5), \mathrm{H}(2.5)$; for the $\mathrm{D} 2$ the relative composition is $\mathrm{C}(50), \mathrm{H}(50)$. The dimensions of the $\mathrm{D} 1$ deuterated scintillator are $\mathrm{R}=2 \mathrm{~cm}, \mathrm{~A}=12.5 \mathrm{~cm}^{2}$ and for the ordinary $\mathrm{D} 2$ plastic scintillator $\mathrm{R}_{\text {outer }}=35 \mathrm{~cm}$, $\mathrm{R}_{\text {inner }}=5 \mathrm{~cm}$. The D1-D2 distance in Fig. 2 is $160 \mathrm{~cm}$, although this parameter has been varied in the optimization of the instrument.

\section{Kinematic cuts}

Neutron spectrometers for fusion need to have a high count rate capability (MHz event rates in D1) in order to deliver useful data with adequate time resolution and small statistical uncertainty. This puts strong demands on the geometrical arrangement and signal processing electronics of the system. With traditional analogue electronics and first-generation digital acquisition systems such rates could only be achieved for a restricted number of experimental parameters - most importantly the time of each event in D1 and D2. With only time information available in high count-rate experiments, a large background of accidental (non-correlated) neutron events will be present when forming time-of-flight spectra from the data.

State-of-the-art digital electronics now makes it possible to record the waveform of each event in the detector system with good absolute timing. This makes it possible to extract correlated time and energy deposition information - kinematics requirements can then be imposed on these correlations that match the physics of the situation.

We have previously, in collaboration with industry ${ }^{2}$, developed and implemented such fast digitizers for the TOFOR system on JET (TOFu) [3] and shown their capability in reducing the accidentals (randoms) background. Comparison with experimental data from the TOFOR/TOFu system has shown that the function of the fast digitizers can be reproduced in great detail in the MCNP simulations of the system. The bTOF system modeled here is equipped with such fast digitizers - the JET experience gives us confidence in the results.

Specifically for the bTOF case:

- A 14-MeV neutron backscattered off a deuteron at $180^{\circ}$ will have $\mathrm{E}_{\mathrm{n}}=1.6 \mathrm{MeV}$ and generate a $\mathrm{D}$ recoil of $\mathrm{E}_{\mathrm{D}}=12.4 \mathrm{MeV}$.

- Based on n,d scattering kinematics and considering also geometry and scintillator energy resolution, we can then impose the requirements on the energy deposition in the D1 detector in order to predominantly select single n,d scattering events:

$$
11[\mathrm{MeV}]\left(\frac{t_{T o F, 14}}{t_{T o F}}\right)^{2}<E_{d e p, D 1}<14[\mathrm{MeV}]\left(\frac{t_{T o F, 14}}{t_{T o F}}\right)^{2}
$$

\section{Performance optimization}

We have previously reported on preliminary results from this study [4]. In the present work we have focused on the optimization of a bTOF instrument with high energy resolution, namely about $\mathrm{dE} / \mathrm{E}=2 \%(\mathrm{FWHM})$. This performance is more than sufficient for DT operations and gives room for relaxing the design if so required by e.g. interfacing constraints. To find the best detection efficiency for this fixed resolution we have varied the thickness of the D1 layers in the range 0.3-2.0 $\mathrm{cm}$ and the D1-D2 distance in the range $60-280 \mathrm{~cm}$, while keeping other geometrical parameters fixed, including a D2 single layer of $4 \mathrm{~cm}$. Note in Fig 3 that the resolution and efficiency tend to have similar dependencies on these parameters. An optimal

\footnotetext{
${ }^{2}$ Signal Processing Devices, Linköping, Sweden
} 
point was found for a D1(i) thickness of $1.6 \mathrm{~cm}$ (total $t(D 1)=8 \mathrm{~cm})$ and a D1-D2 distance of $220 \mathrm{~cm}$. This gave $\mathrm{dE} / \mathrm{E}(\mathrm{FWHM})=\mathbf{2 . 0} \%$, with an optimal efficiency $=\mathbf{0 . 0 0 0 3}$. (Note that in Fig. 3 a correction for the multi-layered structure of D1 is missing - this improves the resolution by about a factor of 3$)$.

a) Resolution (1 sigma) $(\%)$

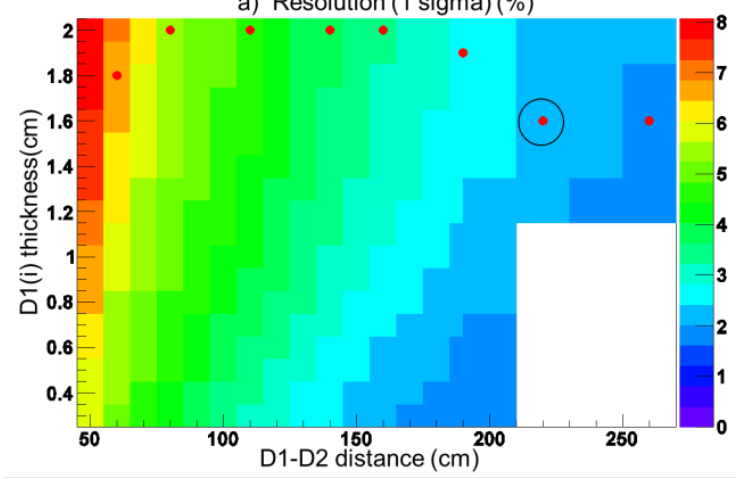

b) Efficiency

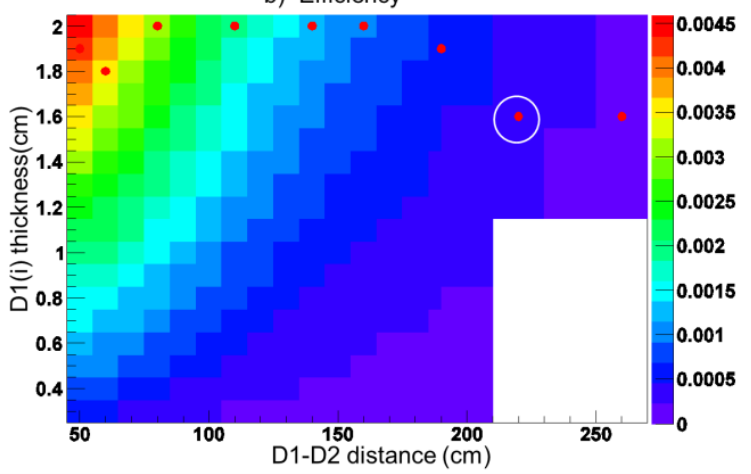

Fig. 3 Results from the optimization calculations. An optimal point is marked.

\section{Response to mono-energetic neutrons}

Figs 4 and 5 show results from MCNP simulations using the optimal bTOF geometry $(\operatorname{dist}(D 1-D 2)=220 \mathrm{~cm}, \mathrm{t}(\mathrm{D} 1)=5 \mathrm{x} 1.6 \mathrm{~cm}, \mathrm{t}(\mathrm{D} 2)=4 \mathrm{~cm}$, etc.) for incident mono-energetic neutrons of $14.0 \mathrm{MeV}$. The nicely peaked (Gaussian) shape of the main single-D scattering response at $\mathrm{t}(\mathrm{TOF})=130 \mathrm{~ns}$ is clear from these simulations, corroborating the assumption that the bTOF is a promising technique for detailed neutron spectroscopy.

In Figs 4 (left, mid) (log scale) are shown the spectrometer's response in terms of time-offlight spectra. In panels a) and b) are shown results without random event effects included in order to emphasize the different physical scattering processes involved. $50 \cdot 10^{6}$ neutrons were launched onto the D1 front surface. Only D1-D2 coincidence events are included. The main peak at $130 \mathrm{~ns}$ is dominated by single elastic n,d backscattering. However, a number of background processes contribute coincidence events under the peak on a level of $10 \%$ and below, as shown in Fig 4(left). These include events involving multiple scattering on Deuterium (red), scattering involving Carbon (green at about 5\%) and scattering involving hydrogen (proton) (blue at less than $1 \%$ ). In general, a background level at about $2 \%$ of the single-D peak intensity is present over a wide range of $\mathrm{t}(\mathrm{TOF})$ values due to multiple scattering off $\mathrm{D}, \mathrm{C}$ and $\mathrm{H}$.

In Fig. 4 (mid) is shown the dramatic improvement achieved when the kinematic cuts are imposed on the data in Fig 4a). In green is shown the t(TOF) distribution of those events that survived the cut - compare to the purple line in the left panel. For comparison the (in simulations) known distribution of true single-D scattering events is shown in blue. Clearly all background events on the low t(TOF) (high energy) side of the single-D peak are removed by the cut, and only a background on the \% level or less remains on the high t(TOF) side. This shows the power of the kinematic cut in this application and the very clean response function in particular on the short time-of-flight side (high energy) - will facilitate analysis of effects due to fast ions.

Fig 4 (right) (linear scale) shows simulation results to illustrate the effect of random (noncorrelated) events in the $\mathrm{t}(\mathrm{TOF})$ spectra. The red curve shows the situation when D1-D2 
coincidences have been formed based on time information only. A rather "poor" signal-tobackground of 1:1 is achieved, which normally would have indicated that the instrument is close to its maximum count rate capability for providing high quality information.

However, applying the kinematical cut (green curve) changes the situation drastically, removing a large part of the random (and other) background, and retaining almost only true single-D scattering events (blue curve). The very small number of background events (green) to the left of the 130-ns peak are randoms due to hydrogen $(\mathrm{H})$ multiple scattering. The high-t(tof) background events are a mix of actual, physical scattering events involving $\mathrm{H}$ and $\mathrm{C}$ (see Fig 4(mid) and some remaining randoms.
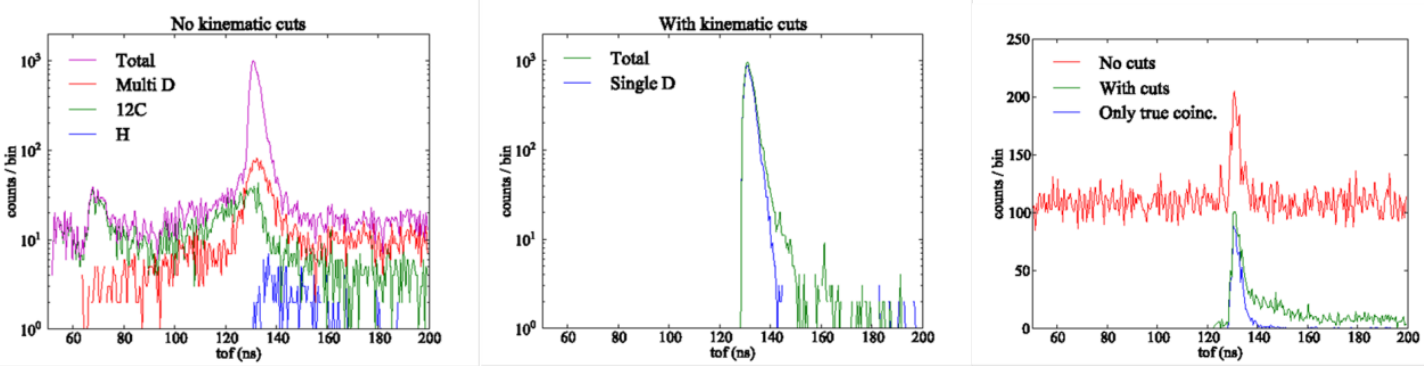

Fig. $4 t_{\mathrm{TOF}}$ spectra from bTOF simulations at the optimal point, $\mathrm{E}_{\text {in }}=14 \mathrm{MeV}$. [left] No randoms, Without kinematic cuts; [mid] No randoms, With kinematical cuts; [right] All events incl. randoms (red); after kinematic cuts (green); true single-D backscatter (blue).

\section{Full response matrix}

In Fig. 5 is shown an example of a full response matrix for a specific bTOF geometry (NOT the optimal point above) as calculated with the MCNPX simulation model described above for neutron energies in the range $2-20 \mathrm{MeV}$. The results are shown after subtraction of random events. In the left panel is shown the situation after a traditional subtraction of a uniform level of random events (see Fig. 4 (right) red curve). To the right is shown the result when kinematics cuts are applied. The intense "orange” band corresponds to neutron single-scattering on $\mathrm{D}$. The first band at low intensity to the right includes the first excited state in C. The more diffuse band at even longer $\mathrm{t}(\mathrm{TOF})$ corresponds to scattering in the surrounding structures.
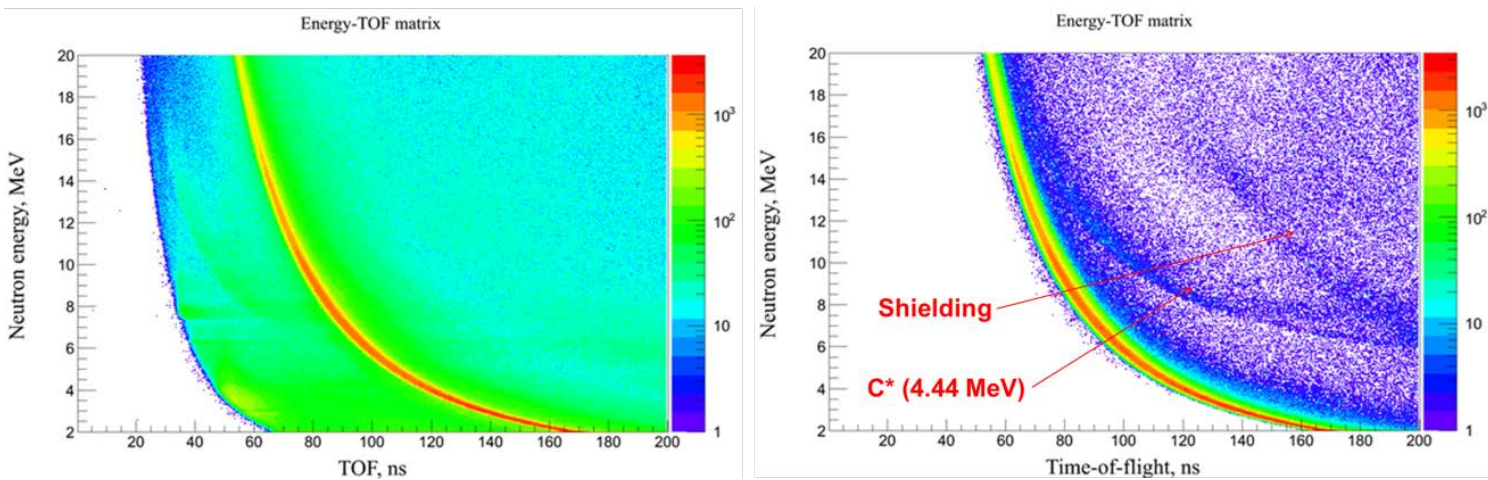

Fig. 5 Example of a simulated, full response matrix for a bTOF with d(D1-D2)=110 cm. For incoming neutrons in the range $2-20 \mathrm{MeV}$. (left) Result after traditional subtraction of randoms;

(right) Result after kinematical cuts 


\section{Discussion}

The work presented here is preliminary. Several details remain to be studied in order to finalize a suitable design for ITER:

- The optimization should be continued by studying also other geometric parameters, in particular D1 and D2 thickness, size and segmentation.

- The aspect of sustainable count rate needs to be considered - the present dimensions of the D1 detectors might need to be reconsidered.

- The found optimal D1-D2 distance might be too large for ITER applications - a conceptual design of a system of $n$ spectrometers for ITER is on-going as an F4E contract and will put boundary conditions with respect to size, weight etc.

- The present results indicate that it will be possible to find a bTOF design with $\mathrm{dE} / \mathrm{E}<$ $5 \%$ (FWHM) of smaller size and sufficient efficiency.

\section{Conclusions}

An optimal design of a high-resolution bTOF spectrometer has been presented based on a double-scattering design with a deuterated first scatterer (D1) and an ordinary second scattered scintillator (D2). The driver in the present design effort has been an energy resolution $\mathrm{dE} / \mathrm{E}$ (FWHM) of about 2\% which is deemed more than sufficient for ITER, leaving room for relaxing the optimized geometrical design if required from interfacing requirements.

The present optimal design has a D1-D2 distance of $220 \mathrm{~cm}$, a deuterated D1 of 5 layers, each of $1.6 \mathrm{~cm}$ thickness, and a monolithic D2 of $4 \mathrm{~cm}$ thickness. The radius of the D1 is 20 $\mathrm{mm}$, and of the D2 $350 \mathrm{~mm}$, the latter with a $50 \mathrm{~mm}$ radius hole to let the collimated $\mathrm{n}$ beam through unhindered. The efficiency of the design is 0.0003 coincidences per incoming $14-\mathrm{MeV}$ neutron, giving a count rate of $300 \mathrm{kHz}$ for a flux of $1 \cdot 10^{9} \mathrm{n} / \mathrm{s}$ impinging on the $12.5 \mathrm{~cm}^{2} \mathrm{D} 1$ front face. Fluxes of this order and higher are expected for ITER DT operations and the expected signal count rate would be sufficient for most spectroscopy functions.

Further work on the bTOF design will include single-detector count rates, optimizing D1 and D2 radii and other geometrical aspects, as well as introducing ITER interfacing aspects into the design.

\section{References}

[1] M. Gatu Johnson et al. "The 2.5-neutron time-of-flight spectrometer TOFOR for experiments at JET”, Nucl. Inst. and Meth. A 591(2008) 417-430

[2] J. Källne et al. "Measurements of the Burn-Up of fast ${ }^{3} \mathrm{He}$ and ${ }^{3} \mathrm{H}$ ions in deuterium plasmas”, Phys. Scripta, Vol. T16 (1987) 160-170

[3] M. Skiba et al. "Fully digital data acquisition system for the neutron time-of-flight spectrometer TOFOR at JET”, Rev. Sci. Instr, Vol 83 (2012) 10D907

[4] C.Hellesen et al., "Prospects for measuring the fuel ion ratio in burning ITER plasmas using a DT neutron emission spectrometer” Rev. Sci. Instr, Vol 85 (2014) 11D825 